\title{
Distributed Knowledge Repositories for Pan-European Public Services
}

\author{
Otmar Adam, Dirk Werth, and Fabrice Zangl \\ Institute for Information Systems (IWi) \\ at the German Research Centre for Artificial Intelligence (DFKI) \\ Stuhlsatzenhausweg 3, Bld. 43.8 \\ 66123 Saarbrücken \\ Germany \\ \{adam, werth, zangl\}@iwi.uni-sb.de
}

\begin{abstract}
The free movement of persons is a concept that has been settled on a European strategic level. In reality there are still a lot of steps to be followed in order to achieve this ambitious goal of mobility. Both the complexity of and the problems encountered in pan-European administrative processes interfere this aim. Interferences can be Knowledge Management (KM) specific (decentralization, implicitness, non-reusability, creation of process knowledge) as well as KM non-specific problems (linguistic, legal, document handling, cultural problems).

To solve these problems both administrative processes have to be made transparent to the citizen and the knowledge about these processes has to be managed. Thus, public administrations must interact seamlessly vertically (Europe, nation, region, municipality) as well as horizontally (between countries) with each other. This implies not only the use of standards but also the interoperability of process systems. Coping with the above mentioned problems a solution requires Knowledge Management. As public administrations are in strongly heterogeneous legal environments a centralised and harmonised solution is not feasible.

In this article a possible solution is described that has been developed in the European research project "InfoCitizen". Within InfoCitizen a Distributed Knowledge Repository and an intelligent agent-based Architecture is identified as an appropriated approach. InfoCitizen is a "proof-of-concept" project in which a Distributed Knowledge Repository and an agent platform are core concepts. The experiences and intermediary results of the successfully ongoing project are presented.
\end{abstract}

\section{Citizens' Mobility in Theory and Practice}

In 1985 free movement of persons was decided in the Schengen Agreement. Being integrated into the EU Treaty with the Treaty of Maastricht in 1993 it aims at increasing the mobility of work forces inside the European Union (EU). [1] This enhanced mobility should improve the flexibility of the employment market inside the EU. The lack of mobility of work forces causes significant macroeconomic damages on the employment market and the current situation should therefore be improved. [2]

The original version of this chapter was revised: The copyright line was incorrect. This has been corrected. The Erratum to this chapter is available at DOI: 10.1007/978-3-540-44836-5_33

M.A. Wimmer (Ed.): KMGov 2003, LNAI 2645, pp. 1-12, 2003.

(C) Springer-Verlag Berlin Heidelberg 2003 
Hence, an effort must be done to reduce those barriers hindering the mobility of persons within the European Union. One of these barriers is the high variety of procedures and responsibilities for similar services, e.g. the delivering of a birth certificate. As everyone has already experienced, it can be quite difficult to receive such services within one country. A certain amount of documents has to be submitted to the administration and the result of the service obtained might have to be forwarded to other administrations. As such chains of processes can be difficult to follow in the own country, it often becomes almost impossible to do so across borders. The consequence is an impediment to international mobility of persons and "imprisons" people in there own country in terms of employment. Furthermore, the current proceeding allows errors in the process since the citizen is also bound to transport documents from one administration to another and to know about his/her duties involved in submitting such documents to an administration. [3] In order to overcome this problem public services and processes have to be integrated.

The knowledge about these processes is dispersed over several administrations, if available at all. Budgetary funds of public administrations are getting shortened every year and yet there is still a problem of public finances. Reducing costs of administrative processes therefore becomes a necessity. An efficient Knowledge Management [4] of administrative processes can not only help to make cross-border mobility easier. It also helps to reduce the costs of these processes.

\section{Process Knowledge as an Enabler for Pan-European Interoperability}

\subsection{Basic Considerations}

Knowledge Management is the creation of value or respectively reduction of costs by an organization (here public administrations) from their knowledge-based assets. The value adding or costs reduction can be as much on a microeconomic level as on a macroeconomic level. On a microeconomic level travel costs can be reduced because the citizen can obtain the same service without transporting paper documents from one administration to the other. Furthermore prices for public services could be lowered, if the internal operating expenses are cut off. On the same level costs can be reduced for public administrations by more time and resource efficient processes. On a macroeconomic level the costs are reduced by a higher efficiency in the working population's mobility and a more balanced budgetary structure concerning the costs of public administrations.

Concretely Knowledge Management means the creation, dissemination and utilization of that knowledge. [5] How the creation, dissemination and utilization of Knowledge can be achieved in Public administrations will be shown in the third section of this paper. Knowledge Management is often assimilated to the use of Information Technology. That is not necessarily true. Nevertheless, Information Technology can be helpful as a supportive tool for Knowledge Management. It will also be shown in this paper how Knowledge Management can be achieved on a strategic business level. Only after this analysis has been done, the possibilities of the support from Information Technology to Knowledge Management in the creation, dissemination and utilization will be covered in detail. 


\subsection{Scope of Problem}

For a successful use of Knowledge Management in cross-border interaction between public administrations several problems have to be solved. A major problem is the fact that process knowledge is distributed, tacit and only shortly gathered with the citizen. Hence it is not accessible and reusable for the process executing administration. The knowledge required to execute is held decentralized, redundant and unstructured on various types of systems. Even within a country the process knowledge is not necessarily available in a structured manner. Figure 1 shows the AsIs-situation and its resulting problems described above. Currently the citizen goes to the employee of a Public Administration (PA) and asks for a service. The PA employee implicitly knows about the partial service (process segment) he has to execute, the input(s) he needs to execute the service (e.g. a certificate of birth) and the outputs that the service provides (e.g. a marriage notification). It is yet the task of the citizen to know where to get the required input and where to bring the output produced by the service that he requires. Furthermore the input and output providers will again implicitly know about the process segments that they have to execute. Summed up, on one hand it is the citizen who knows for the duration of the service provision what process segments have to be executed and who can execute them. On the other hand it is the Public Administrations as well as the input and output providers who know how to execute the process segments. After the service has been provided the knowledge of process segments sequence and its executor is kept by the citizen and therefore is not reusable. This shows that knowledge is decentralized and implicit.

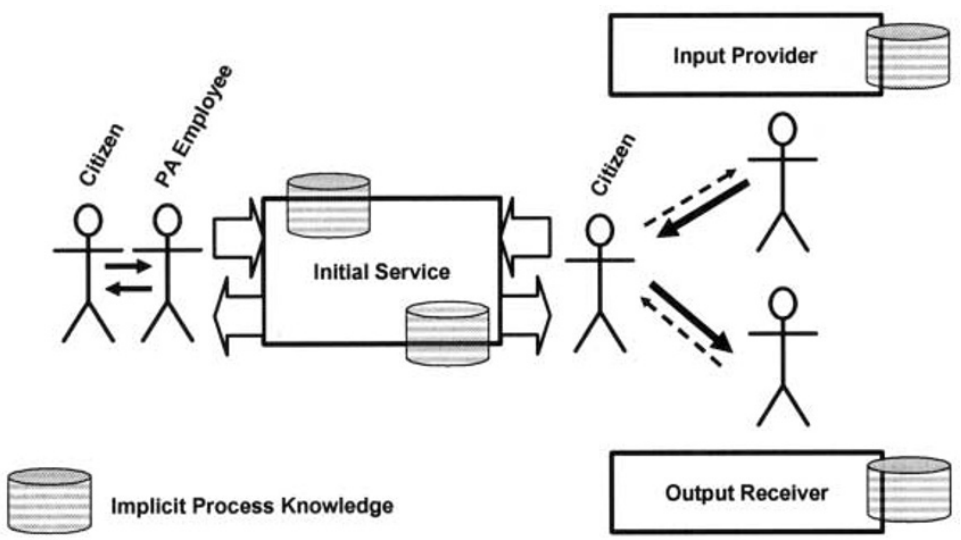

Fig. 1. As-Is-situation in public administration's procedures

Figure 1 shows the present situation where the citizen has to start every single public service himself. The citizen gets information from the public administration employee on the documents required to fulfil the initial service. The citizen then gets these documents from other second level public services that again might need some additional documents to be accomplished. The knowledge on required documents for the initial service is distributed between the concerned public administrations and the citizen is in charge of the transport of the documents as well as the process order, i.e. the public service to start in order to get the documents. 
Apart from the Knowledge Management specific problems in public administrations a citizen can encounter further problems when executing an international process. The language in which the required documents (inputs) or out coming documents (outputs) are written is most likely to differ between one country and another. To resolve that difficulty the citizen has to have the documents translated and the translated documents certified. More over following international processes also implies for the citizen to pull information from many different sources to gather knowledge on how to "build" the process. A further problem can occur when the citizen brings paper-based documents to a public administration and the administration has to enter data manually into its system. Some required information might also be missing on a document from a foreign administration requesting the citizen to bring even more documents. Finally, there also might be legal differences between two or more countries which can make international administrative processes more difficult.

\subsection{Requirements}

In order to remove the above mentioned barriers and problems process knowledge needs to be created, disseminated and utilized [6]. To create process knowledge its knowledge assets have to be selected and documented. However there is too many information to be all taken into account. Therefore methods have to be used or even developed to document (process) knowledge in a formal way and also to enable continuous knowledge growth.

For dissemination purposes (process) knowledge needs to be made available so it is accessible at the right time in the right place. That knowledge also has to be sustainable and accurate over time. Furthermore the knowledge needs to be pushed to concerned entities. This implies that full process knowledge has to be brought to the citizen; if this is necessary at all. A more improved solution would avoid making it necessary for the citizen to acknowledge the process and make the latter completely transparent to him/her. This way the use of public services would be greatly facilitated for the citizen and hence also reduce barriers to (cross-border) mobility.

In order to utilize (process) knowledge efficiently it has to be retrieved methodically from the above mentioned documentation. Thanks to these methods the knowledge would be adapted to the entities using it, hence the knowledge should be citizen or administration aligned oriented.

Finally, knowledge has to be reusable. Therefore it has to be made understandable for any entity accessing it, e.g. a system of a public administration. The reusability of knowledge is a condition for interoperability. If knowledge is stored but cannot be accessed or used by concerned public administrations, interoperability can per se not be achieved. Therefore process knowledge enables interoperability, which is the final condition to cross-administration and cross-boarder process and knowledge management.

Interoperability is the ability of two or more systems to exchange information and to use the information that has been exchanged, as well as the capability of two or more environments to handle the same input (information or service) in the same way. The interoperability can be obtained ether by adhering to public interface standards or by using an agent that converts one system's interface into another. With this understanding of interoperability it becomes evident why first, Process Knowledge 
enables interoperability and second, why interoperability is needed for a platform that intends to support transparent pan-European and cross-administration processes with the exchange of electronic standardized documents. [7]

\section{Process Knowledge for Integrated Public Service Provision}

As described above the main focus of current integration projects is to facilitate the use of public services by the citizen. In order to achieve the demanded transparent service provision the public authorities must communicate and interact seamlessly with each other. Current approaches are mostly limited to standardization of data exchange so that documents are well-defined and can be generated and interpreted unambiguously by each participant. These efforts are necessary but not sufficient to enable interoperability between European Public Administrations. Within the local offices the procedures are analyzed and optimized with process management tools from an intra-organizational perspective and are supported by IT. Considering interorganizational aspects in contrast the local interfaces to procedures in other public administrations are treated manually. To improve this situation for both the citizen and the public administrations an automated execution of cross-level and crossnational procedures must be established.

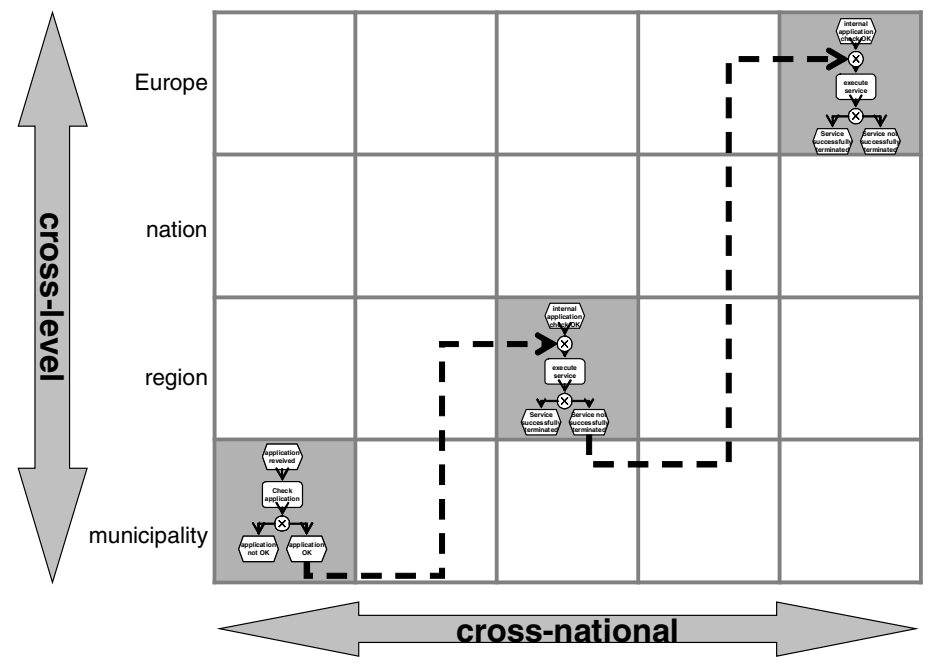

Fig. 2. Interaction of Public Service Offers

To enable this kind of interoperability the local service must retrieve information about services that provide needed inputs and services that process the outcomes at least on a meta-level. The initial service must know the characteristics of these services i.e. the service or process knowledge [8] must be accessible through standardized mechanisms. As the potential impact of managing process and service knowledge is clarified now the needed knowledge management activities are characterized in the following. 
A common segmentation of KM activities distinguishes between the identification of existing knowledge assets, lacks of knowledge and external resources to fill these gaps, the augmentation and documentation of available knowledge and the use respectively reuse of knowledge. [9] This basic scheme can be applied to the management of process knowledge in e-Government scenarios.

Identification:

- Business process modelling and redesign within the local offices is the foundation to document existing procedures. Thus the created and maintained process models represent the knowledge assets of the local offices.

- Undetermined interfaces to other authorities with which the local public administration collaborates vertically and horizontally have to be identified. Thus the missing knowledge is determined.

- Resources for external knowledge acquisition (knowledge base) means black box knowledge about inputs and outputs of external services. The local knowledge is extended by the documents that have to be exchanged in a cross-organizational scenario. A distributed service knowledge repository is potentially able to fill this gap.

Augmentation/Documentation:

- By establishing standardized request operations that regulate the search for appropriate preceding and subsequent services the service instances can look for the input providers and output receivers that are needed to successfully execute a local service.

- At the local service interfaces the retrieval mechanisms must be added and integrated.

- The interaction mechanisms and communication acts are modelled so that the connection to other systems possibly through adequate adapters can be established. One subtask in this context is the standardization of data exchange.

- The knowledge gathered in this stage is static knowledge with a medium- to longterm validity.

Use/Reuse:

- The static knowledge described above is used to retrieve potential input providers and output receivers. The routing of documents is generated dynamically at runtime.

- The dynamic knowledge is cached and reused when the same or a similar business process is executed. This can be seen as a learning component of the system.

As illustrated the process knowledge is to be identified, documented, stored and made available on the right time at the right place. The easiest way would be to set up a central database that holds all the information that is needed. At this point the specifics of the e-government domain have to be taken into account. On a panEuropean level or in a federally structured country no such central database can be established, amongst others because of legal restrictions on personal data protection. Further more there are major performance and reliability concerns considering central IT-systems in decentralized structures. 


\section{Designing Distributed Knowledge Repositories}

The inappropriateness of a central solution arises from the circumstances described above. An alternative solution is the use of concepts that follow the paradigm of decentralization by keeping distributed data. This approach implies that all data that represent routing decisions (concerning input and output) are not maintained centrally but in logically many repositories. These repositories are virtually aggregated and integrated without establishing one central repository. For this purpose intelligent search and retrieval mechanisms are used. As static information the services that are offered by a Public Administration are stored in the local repository and are made accessible. At run-time the retrieval function determines the input providers and output receivers dynamically by accessing the virtually aggregated repository. Thus the business process fragments that are stored as modules in the decentralized on-siterepositories are connected and the customized and integrated overall-process can be executed. This effect is illustrated in Figure 3.

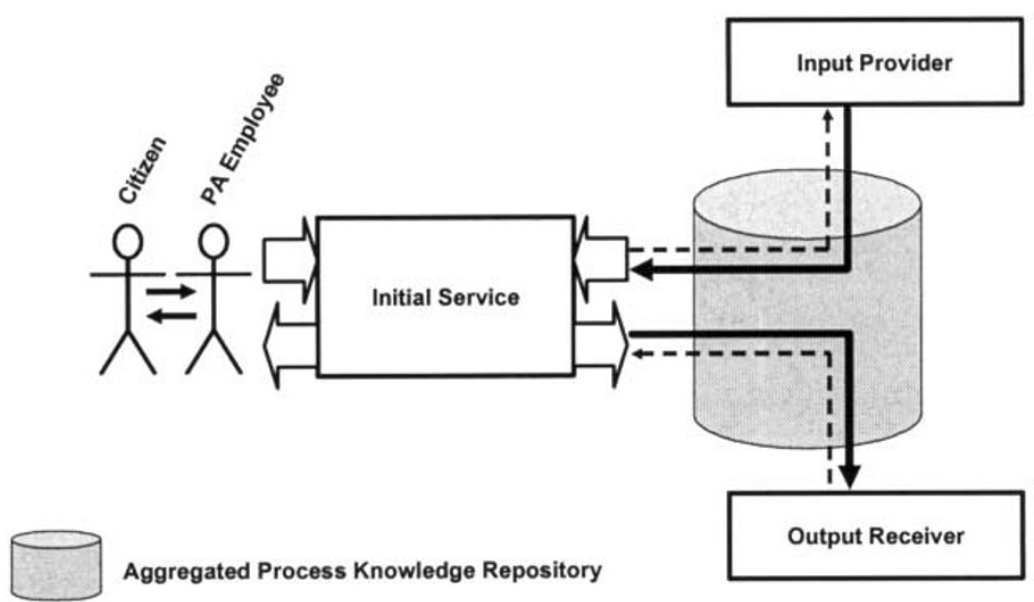

Fig. 3. Process Knowledge Management Repository

This dynamic process customisation can be explained with the following example. A citizen has moved from one country to another. According to the law he has to register the change of residence at the Public Administration of the municipality he has moved to. Additionally he has to deregister with the authorities of his former home country where a national agency is responsible for residential issues. To perform the service "change of residence" the certificate of birth of the citizen is needed. An authority of the region where he was born can issue this document. Without automated service interaction the citizen has to know these interdependencies and get the certificate of birth. At the new registry he hands out the certificate and after checking the documents his change of residence is officially registered. The citizen gets a certification of the new registration with which he or she can go to the former registry and the deregistration is performed. All these activities, especially the exchange of documents, were carried through manually by the citizen. The nonavailability of cross-organisational process and service knowledge causes this lack of 
integration. With the distributed services repository described above the procedure would be customer-friendly. The citizen just moves to the new place and initiates the "change of residence" service. The employee checks the ID of the citizen and triggers the process. The service itself "knows" which inputs are required; in the underlying case this is the certificate of birth. The service retrieves the services that are potentially able to provide this input document as an output by asking the knowledge repository.[10] An intelligent search mechanism or a software agent finds the respective services and returns the list. These services are invoked sequentially by priority order as long as the main service can be successfully executed. This can be called a kind of "pull strategy". After that the output of the main service is delivered to the Citizen. Additionally other outputs have to be distributed to services that need the generated output as initial input. In the described scenario this is the notification about the change which implies the deregistration with the former registry. The so called "push strategy" retrieves a list of services that need this kind of input from the knowledge repository and they are started by delivering the generated output documents. At this point the translation of process knowledge into control knowledge[11] is needed. The static process knowledge has been used to generate the service instance. This instance requires now dynamically composed routing which is specific for the current service. The control knowledge is able to provide that information.

\section{The InfoCitizen Approach}

\subsection{The InfoCitizen Project}

The Project InfoCitizen, funded by the European Commission under the 5th Research Framework Programme, aims to create a pan-European Information Architecture for EPA interoperability as well as to develop specific information technology that supports this Architecture and ensures a seamless information exchange between European public administrations on a pan-European level. Moreover, with this solution EPAs are able to provide transparent and integrated public services for their citizens as well as for external clients.

InfoCitizen started in September 2001 with a project volume of 3.3 million euros for a duration of 24 months. Eleven organizations within five different EU-countries are working together to succeed the challenge of pan-European interoperability. The showcase scenario is tested in the municipalities of Colleferro (Italy), Schmelz (Germany), and Tres Cantos (Spain), and the prefecture of Thessaloniki (Greece).

\subsection{The InfoCitizen European Architecture}

The first major milestone was the development of a generic Interoperability Framework, the InfoCitizen European Architecture. It describes the relations and specifications of knowledge and information exchange between EPAs, i.e. the interPA interaction. The major requirements that were addressed with this Architecture are to support and describe the two key goals of the user needs, namely: 
- transparent public service provision to the European citizen

- multi-agent / multi-country setting of public service provision

The InfoCitizen European Architecture will conduct electronic transactions in multiagent settings/multi-country settings in a transparent as possible manner for the European citizen.

In order to create a long-living and stable result, we decomposed the European Architecture into three parts:

1. The Conceptual Architecture provides a business level view on the solution. It addresses the EPAs and their scope of description.

2. The Technical Architecture transforms the conceptual solution into a system-level architecture that describes the technological system without specifying concrete technology; it is technology-independent and therefore disconnected from the rapid-changing technology layer.

3. The System Architecture describes the system to be build. It is the blueprint of the InfoCitizen software solution based on an appropriate selection of state-of-the-art technologies.

The main challenge of the European Architecture, esp. of the Conceptual Architecture was the management of the control knowledge for the interaction. The central object in this context is the public service provided by a specific EPA. As already explained both the inputs needed for processing the service as well as the outputs produced by the service have to be received respectively distributed to the citizen in a transparent manner. Therefore the service itself has to conduct two different strategies for information retrieval and delivery.

1. The "Pull" strategy means that the required input of a service should be automatically accessed when needed from its relevant source (which is another service).

2. The "Push" strategy means that the consequences of the PA service provided should be "propagated" to all relevant PAs (respectively their appropriate services).

Besides the information exchange problem, that will be discussed later, the difficulty is how to identify the appropriate service. The knowledge for answering these kinds of questions is distributed over the different EPAs. Therefore InfoCitizen uses a distributed knowledge management approach. Each EPA publishes both the services it provides and detailed information about the services, such as mandatory or optional information inputs, outputs and preconditions.

Looking at a single instance of a service provision, it is necessary to find a mapping between services looking for specific information and services able to provide these. These mapping is an individual action, specific for each instance of service provision. As an example a service "marriage" with instance "Man A (German) and Woman B (Italian)", both needing certificates of birth, naturally has to communicate with different services than the instance "Man C (Greek) and Woman D (Spaniard)". To enable this mapping search a service uses the knowledge published by the other EPAs and determines potential information sources and targets. For technical reasons within InfoCitizen we decided not to hold the complete knowledge in each EPA but in a central repository, where they can access it. This is not opposed to our distributed knowledge management concept, as the central storage represents only a simplified communication media.

Obviously, to successfully communicate the services must understand each other. To solve the Babylonian problem of data format mapping a "Common Document 
Language" has been developed. It is an open and extensible specification describing the data to be exchanged within InfoCitizen. It relies on state-of-the-art XML technology and is based on existing standards. Using the "Common Document Language" we provide a flexible instrument to find a common definition of the syntax and semantics of data within EPAs without forcing them to change internally.

\subsection{InfoCitizen Platform}

The information-technological realization of the concepts described uses agenttechnology. This is most suitable for our purposes because it assists and facilitates the process of service search and discovery. Therefore the main component of the InfoCitizen platform is the interoperability agent. It plans controls and executes the information exchange. Using the emerging agent-technology enables the platform to efficiently search for, retrieve and distribute documents in order to satisfy the information demand of the EPAs that are connected to the InfoCitizen platform.

Figure 5 shows the central role of the Interoperability Agent within the structure of the InfoCitizen system architecture.

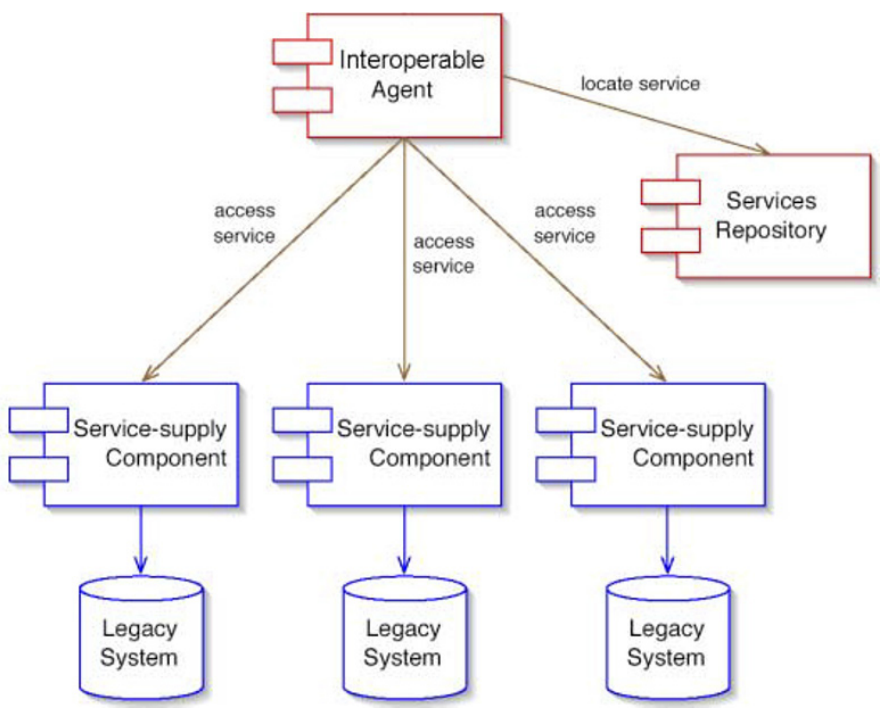

Fig. 4. InfoCitizen System Architecture

We implemented a services repository where all relevant information about services and input respectively output documents are stored. The service information was set up in a distributed manner and updated by the EPA that is responsible for this specific service. When searching for an appropriate service, the Interoperability agent queries and analyses the data of the services repository and finally locates the specific service.

A problem in real scenarios is the heterogeneity of EPAs. Within the context of the electronic support of service transactions triggered from or targeted to external systems, the existing legacy systems of the EPAs have to communicate with the other 
systems and therefore have to be-at least indirectly-connected to them. This connection will be realized through the Service Supply Component, which acts as an adapter between the existing legacy or standard-software application systems of EPAs and the InfoCitizen platform. The Service Supply Component Architecture is a Source code-Framework that simplifies and fastens the implementation of specific Service Supply Components. Moreover we provide precompiled and customisable Standard Service Supply Components that can be deployed instantaneous for widely common applications such as JCA compliant ones or JDBC compliant databases.

The front-end should satisfy user needs in usability and process support. Based on internet-technologies we are developing a multi-lingual, customizable information portal solution that interfaces between the user and the InfoCitizen platform.

In order to prove our concepts and implementations we are setting up a pilot system, which includes

- Local pilot showcases in the four participating countries that will focus on commonalities of business processes,

- and a generalised, pan-European InfoCitizen trial, which will reflect experiences and results in the international information exchange between the particular pilot sites.

Even as this pilot is showing interoperability only between four different European public administrations, we not only evidence the conceptual and technical feasibility of integrating these four specific offices. In view of the general approach we created with both the conceptual ideas and the technical realization, we can show that at least an interoperability between any public administration within these four countries can be established using the InfoCitizen European Architecture and the InfoCitizen Platform.

\section{Conclusion}

As a first result relevant processes in the Public Administrations of the four userpartners were documented and analysed. This serves as a basis for the improvement of existing processes and the invention of new services that facilitate the usage for the citizen. Furthermore generic models for almost any governmental service provision have been deducted by abstraction. These models are integrated in the InfoCitizen European Architecture which offers support for administrative business processes considering standards and interoperability. On this foundation a prototype consisting of locally installed Service Supply Components, an agent platform and a front-end is implemented. To prove the validity and practicability of the developed architecture the prototype is used in the showcase enabling the interaction of EPAs in four European countries. The move, marriage and adoption procedures in multi-country settings can be executed using the InfoCitizen System.

In the long run the InfoCitizen results are intended to serve as reference models for the electronic interaction of any Public Administration and thus establishing costeffective and time-saving interoperability procedures that also improve the service quality considering the citizen. The Services Repository implementing distributed Knowledge Management enables this interoperability between EPAs. But the use of the repository is not limited to this purpose. In order to generate new added value of 
public products and services EPAs need more knowledge. Therefore the content of the repository will grow in quantity and quality to satisfy this future needs. Enabling the public administrations in Europe to provide transparent and integrated services on local, regional, national or pan-European level is the challenge for the future. The InfoCitizen project is a first step towards that future.

\section{References}

1. Fontaine, P.: Europa in zehn Lektionen, in: Europäische Kommission (Hrsg.): Veröffentlichungen für ein breites Publikum, 1997, <URL: http://europa.eu.int/comm/publications/booklets/eu_glance/ 12/txt_de.htm>.

2. Jansen, S. A.; Priddat, B. P.: Electronic Government - Neue Potenziale für einen modernen Staat, (Klett-Cotta) Stuttgart, 2001, S.22-23.

3. Meir, J.: Geschäftsprozesse im eGovernment - Ein Überblick, Arbeitsbericht Nr. 5 des CC eGovernment, Berner Fachhochschule - Institut für Wirtschaft und Verwaltung, Bern, 2002, S. 19-20.

4. Attenda: Government e-enables neighbourhood renewal - Attenda operates online knowledge management system, M2 Presswire; Coventry; Feb 6, 2003.

5. Newman, B.: An open discussion of Knowledge Management, 1991.

6. Kresner, Richard: Building a knowledge portal: A case study in Web-enabled collaborations, Information Strategy; Pennsauken; Winter 2003.

7. Fumio, Hattori; Takeshi, Ohguro; Makoto Yokoo; Socialware: Multiagent systems for supporting network communities, Association for Computing Machinery. Communications of the ACM; New York; Mar 1999.

8. Rolland C.: "Modelling the Requirements Engineering Process", Proc. Fino-Japanese Seminar on "Conceptual Modelling", 1993.

9. Probst, G.; Raub, S.; Romhardt, K; Doughty, H.A.: Managing Knowledge: Building Blocks for Success, John Wiley \& Sons, 1999.

10. Seokwoo, Song: An Internet knowledge sharing system, The Journal of Computer Information Systems; Stillwater; Spring 2002.

11. Wilkins, D.; Jardins, M.: A call for knowledge-based planning, AI Magazine; La Canada; Spring 2001.

12. SAP: Next generation of manufacturing solutions to more tightly link shop floor with supply chain networks; Development resources dedicated to design solutions that will reduce working capital requirements and improve asset turns and cycle times, M2 Presswire; Coventry; Oct 29, 2002;

13. DATA PROTECTION REGISTRAR: Data Protection Registrar welcomes proposal for information commissioner, M2 Presswire; Coventry; May 26, 1999. 